\title{
Le riz au Japon, au regard de la culture, de la langue et du droit
}

\author{
Yuki Horie $^{1}$ - Béatrice Crane-Mikołajczyk ${ }^{1}$
}

Published online: 21 August 2019

(c) The Author(s) 2019

\section{Résumé}

Le riz est fondamentalement un produit, une notion, et un droit garanti au Japon. Son approche est complexe, variée, étendue, tant du point de vue culturel, linguistique que juridique. Son rôle dépasse largement le cadre nutritionnel, quoique comme le blé en Europe, il reste un aliment essentiel dans l'art culinaire japonais, par tradition, par habitude et de par la loi. Si l'origine du riz est chinoise, après son introduction au IV ${ }^{\text {ème }}$ siècle av. JC, il est devenu un, ou même, le symbole de la culture japonaise; cela se perçoit jusque dans la langue, qui lui accorde un statut particulier, singulier par rapport aux autres aliments. Ceci se retrouve même dans le droit japonais, ancien ou contemporain.

Mot clés Origine légendaire du riz japonais · classification du riz · culture alimentaire $\cdot$ loi sur les aliments $\cdot$ réglementation des appellations

\section{La culture du riz}

Avant de traiter de la culture du riz, il nous faut remarquer que l'histoire du riz dans la société japonaise relève du légendaire ${ }^{1}$ car il était considéré par les gens, qui vivaient à l'époque où l'écrit n'existait pas, comme un «produit donné» par les

\footnotetext{
${ }^{1}$ En effet, le riz ne peut pas être considéré comme un simple aliment. La graine présente des origines mythico-religieuses, elle fait partie intégrante de bon nombre de cérémonies religieuses, elle occupe une place centrale dans les rites de succession impériaux, elle rime traditionnellement avec prospérité, et a pu, sous sa forme d'aliment cuit, être considérée comme une métaphore de l'unité familiale [1]. Comme le pain pour la France et les Français, le riz constitue un élément majeur de la culture traditionnelle japonaise. De manière plus ou moins consciente, les menaces qui pesaient alors sur le riz furent fréquemment ressenties comme des menaces pesant sur un élément constitutif essentiel de l'identité collective [2].
}

Yuki Horie

pupu720205@gmail.com

Béatrice Crane-Mikołajczyk

beacrane@amu.edu.pl

1 Université Adam-Mickiewicz de Poznan, Poznan, Poland 
divinités. Dans les mythes et les histoires dans la vieille tradition japonaise, il est écrit que le riz est venu du monde des dieux : «il y a longtemps, au-dessus de notre monde, il y avait le monde d'un dieu et l'enfant de celui-ci qui y vivaient avant de tomber dans ce pays». Le nom de cet enfant était Hō no Ninigi no Mikoto. Hō veut dire «les oreilles de riz»; Ninigi veut dire «vivant», c'est-à-dire que «le riz pousse beaucoup grâce à dieu». La grand-mère de Hō no Ninigi no Mikoto était la déesse du soleil qui s'appelait Amaterasu no ōmikami (天照大神). Amaterasu no ōmikami donna le riz à son petit-fils lorsque le Hō no Ninigi no Mikoto tomba sur le sol, en lui disant qu'il devait faire pousser beaucoup de riz dans le monde entier. C'est pour cela que les personnes à l'époque considéraient que le riz était une chose précieuse qu'ils avaient reçue de Amaterasu no ōmikami. Partant de l'origine acceptée dans l'inconscient culturel, nous entrevoyons déjà la richesse de la valeur du riz.

\subsection{La culture du riz: les conditions propices à ce type d'agriculture}

Si le riz est un aliment qui convient au goût des Japonais, il a l'avantage de pouvoir être conservé longtemps. Consommable tout de suite après la récolte, il peut être gardé pour plus tard encore en cas de pénurie. De plus, le climat est bien adapté pour la culture du riz [1].

Les conditions climatiques au Japon sont optimales pour cultiver le riz; en commençant par la température élevée et l'humidité : il pleut beaucoup pendant la saison des pluies et au début de l'été. Ensuite, pour le fleurissement et l'apparition des oreilles de riz, ${ }^{2}$ le rayonnement solaire (la quantité et le temps d'ensoleillement) est suffisamment fréquent. Enfin, de belles journées d'automne se suivent assez longtemps pour parfaire la pousse jusqu'à la récolte.

Notons que le climat chaud et humide, pluvieux en été et neigeux en hiver, est en effet propice pour la culture du riz, mais il n'est pas approprié pour le blé, aliment de base de la civilisation occidentale. Le blé est semé à l'automne et récolté au début de l'été, après une croissance vigoureuse à la faveur du printemps. Tout d'abord, l'amplitude des températures au Japon, soit la différence entre les périodes de chaleur et de froid, plus que toute autre chose, est trop grande pour la culture du blé. L'hiver est trop froid, et durant le stade de croissance en été, il fait trop chaud. Aussi, en hiver, le blé a besoin d'une certaine quantité de précipitations, mais pas de neige. Le blé couvert de neige ne peut pas pousser correctement. Alors que le climat sec est nécessaire au temps de la récolte, juste à ce moment-là au Japon, il y a la saison des pluies.

\subsection{Les régions où le riz est cultivé}

Il y a de nombreuses régions qui cultivent le riz telles que Hokkaido et les préfectures de Niigata, Akita, Fukushima, Yamagata. Niigata et Akita sont connues comme Komedokoro, les régions les plus prospères de la culture de riz, depuis les temps

${ }^{2}$ Les épis. 
anciens, ou encore Koshihikari de la préfecture de Niigata. La ville Uonuma est devenue célèbre pour son riz considéré comme le meilleur du Japon. Hokkaido, en raison de ses basses températures, n'était pas considéré auparavant ${ }^{3}$ comme convenable pour la culture du riz. Néanmoins, des variétés poussant dans le froid ont été développées comme les riz appelés Kirara 397, Hoshino Yume et Nanatsu Boshi.

\subsection{Les travaux agricoles de la culture du riz... chaque travail a son nom}

La culture du riz implique de nombreuses étapes et ainsi exige un calendrier précis pour parvenir à sa récolte. Les conditions climatiques et géographiques ne sont donc qu'un préalable pour exécuter les travaux agricoles.

(i) En avril, 稲づくり inezukuri:

La culture du riz commence au printemps. Tout d'abord, on fait tremper les graines dans l'eau. Après, on recouvre les graines d'une mince couche de sol pour les réchauffer dans une serre et attendre leur germination.

(ii) En mai, 田おこし taokoshi:

Il faut creuser finement le sol et mettre de l'engrais dans les champs de riz. Ensuite, il faut ajouter de l'eau et remuer le sol. L'eau est prélevée par des canaux d'irrigation.

(iii) En juin, 田植えtaue:

C'est la période pour planter des semis de riz dans les champs de riz remplis d'eau. La plantation est le travail le plus important parmi les travaux agricoles. Des événements traditionnels sont organisées dans diverses régions après la plantation du riz.

(iv) En été:

Il faut enlever les mauvaises herbes ou mettre de l'engrais.

(v) En septembre, 稲刚り inekari; もみすり momisuri:

On récolte avec une moissonneuse-batteuse. Il faut ensuite sécher les plantes en ne prenant que le riz (sapin) du brochet. Et enfin, il faut enlever la paille du riz brun.

\section{La cuisine et les valeurs coutumières du riz}

\subsection{La notion de shushoku (主食): aliment de base}

Le riz au Japon est appelé «aliment de base». La notion d'aliment de base signifie non seulement un aliment qui est la principale source nutritive, mais cela traduit aussi une pensée affectueuse ${ }^{4}$ pour le riz japonais. Il fait partie intégrante de la

\footnotetext{
3 Le premier essai de culture du riz à Hokkaido date de 1873.

${ }^{4}$ Cette importance se distingue même à partir de la fin du VII ${ }^{\mathrm{e}}$ siècle après la réforme de Taika jusque vers le $\mathrm{X}^{\mathrm{e}}$ siècle, à l'époque Ritsuryō, moment où le riz (comme la houe, le sel, etc.) devient une valeur monétaire pour le paiement des impôts.
} 
culture, de la mentalité locale, car il est considéré comme un véritable et pur produit du pays, cultivé sur une longue période de l'histoire.

D'après le ministère de l'Agriculture, des Forêts et de la Pêche, les aliments de base sont la nourriture que les gens mangent le plus souvent au quotidien. En général, en Europe, les céréales et les pommes de terre sont des aliments de base typiques. Cela veut dire également que les producteurs de riz sont normalement plus riches que d'autres agriculteurs, de même que la stabilité de la production est plus grande par rapport à d'autres cultures. Le contenu du repas japonais est caractérisé par un bon équilibre entre les nutriments nécessaires, donc par la répartition et la combinaison avec l'aliment de base des plats principaux et des accompagnements.

Les aliments de base sont en général le riz, le pain, les céréales, les pâtes, et sont notamment une source d'énergie en glucides. Les plats principaux peuvent être le poisson, la viande ou les œufs, c'est-à-dire principalement des sources de protéines et de lipides de haute qualité. Ils deviennent le centre d'un plat, aux côtés d'accompagnements pouvant être du soja, des légumes, etc., qui apportent des vitamines et des minéraux, et des éléments au rôle important comme les fibres alimentaires.

Mais pourquoi existe-t-il ce rapport spécial des Japonais au riz et ce sentiment qu'il est un aliment de base, au-delà d'être une source d'énergie? Jusqu'à l'ère Edo, ${ }^{5}$ le classement de chaque clan était évalué par kokudaka, c'est-à-dire la quantité de riz produit. A cette époque-là, la culture du riz était au centre de l'agriculture. Cependant, ceux qui produisaient le riz, le plus souvent, ne mangeaient pas de riz. Ils mangeaient des céréales, des pommes de terre et des légumes. Le riz était trop précieux pour les paysans.

Dans les zones rurales, seuls les habitants qui avaient un statut social élevé, ou qui étaient riches, pouvaient manger du riz. Pour les agriculteurs, le riz blanc était une nourriture spéciale qu'ils pouvaient seulement manger pour des occasions particulières, appelées hare comme le Nouvel An et certaines fêtes.

Bien qu'il ne soit pas complètement certain que ce soit à cause de ce qui est évoqué précédemment, c'est un fait que les Japonais témoignent d'un sentiment exceptionnel pour le riz. Le Japon, auquel on a associé très souvent l'expression «nation de Beishoku» (manger du riz), a en vérité plutôt été jusqu'à récemment la «nation qui désire le Beishoku». Cet avis est partagé par nombre de chercheurs. ${ }^{6}$ Nous admettons que le riz joue un rôle économique et culturel de grande importance dans

\footnotetext{
5 C'est-à-dire le début du XVII ${ }^{\mathrm{e}}$ siècle (1603-1868).

${ }^{6}$ Ce point de vue est partagé par Emiko Ohnuki-Tierney [3] qui étudie d'un point de vue ethnologique l'importance du riz au Japon. Il souligne le fait que si les Japonais s'associent avec le riz, cela ne veut pas dire qu'obligatoirement ils en étaient de grands mangeurs: «A study of rice as a metaphor of the Japanese self, then, involves the question of how rice has become a dominant metaphor of the Japanese despite the fact that a large segment of the population has always been engaged in occupations other than rice agriculture and, as mentioned, rice has not been a quantitatively important source of food for a large segment of the Japanese population.» p. 19. Une étude du riz en tant que métaphore des Japonais eux-mêmes implique alors la question de savoir comment cela s'est établi malgré le fait qu'une large part de la population a toujours été engagée dans des occupations autres que la culture du riz. En effet, comme cela a été mentionné, le riz n'était pas quantitativement une source importante de nourriture pour une grande partie de la population japonaise.
} 
plusieurs sociétés asiatiques, à tel point que chīfàn (吃飯), makan nasi, kin khao en chinois, indonésien, thaï, etc. se traduiraient par «manger» en général ainsi que «manger du riz» en particulier. Mais il nous semble aussi que cette notion d' «aliment de base» concernant le riz est particulièrement profonde au Japon.

Si le riz est considéré comme la nourriture la plus essentielle, il doit être fourni de manière stable. Au Japon, en plus de l'importance des aspects quantitatifs, le climat approprié pour la production de riz lui apporte son excellente valeur nutritive (l'amidon est lentement digéré; l'équilibre des acides aminés et des vitamines est vanté). De plus, dans la culture alimentaire, le riz se situe au centre.

\subsection{Un rôle d'ambassadeur et de propagation de la culture japonaise}

$\mathrm{Si}$, dans les pays occidentaux, il y a de plus en plus de restaurants japonais, en particulier pour les amateurs de sushi, on note un boom de la nourriture japonaise en général partout dans le monde. La nourriture japonaise, bonne pour la santé, délicieuse à manger et magnifique à regarder, s'est propagée dans les pays étrangers, bien qu'aux États-Unis, en Europe, et dans plusieurs pays asiatiques beaucoup de restaurateurs soient chinois et que la cuisine soit souvent adaptée aux goûts locaux. Les endroits où il y a de nombreux restaurants japonais sont soit ceux qui ont une histoire familière avec la nourriture japonaise, comme la Corée du Sud, soit ceux où la présence d'expatriés et de résidents japonais est importante tels que New York, la Californie, Bangkok ou Sao Paulo.

En Californie, il y a beaucoup d'Américains d'origine japonaise, des descendants de migrants de la deuxième génération, et c'est pour cela que l'Etat est appelé «le lieu de naissance de la nourriture japonaise aux États-Unis». Il y a de nombreux restaurants japonais sur place. Le sushi est la nourriture la plus populaire pour de nombreux Américains. Parmi les restaurants japonais à New York, plus de $60 \%$ proposent des sushis dans leur menu. La nourriture japonaise est également populaire en Europe. Le nombre de restaurants japonais à prix raisonnables, dont les sushis bars, y a considérablement augmenté. Environ $70 \%$ des restaurants japonais proposent des sushis en Europe. Selon une enquête menée par JETRO en décembre 2013, qui ciblait 3000 étrangers de 10 à 50 ans dans six villes (Moscou, Ho Chi Minh-Ville, Jakarta, Bangkok, Sao Paulo, Dubai), la plupart des gens ont cité comme plats connus le sushi et le sashimi $(35,3 \%)$, le tempura $(9,6 \%)$ et le yakitori $(8,7 \%)$. Le sushi, qui occupe la première place, est surtout considéré comme un repas sain. La balle de riz onigiri est aussi devenue célèbre en Europe. En outre, certains n'en ont jamais mangée, mais l'ont vue dans les films d'animation japonaise.

En outre, l'Organisation des Nations Unies pour l'éducation, la science et la culture (UNESCO) a érigé le washoku (la nourriture japonaise) en patrimoine culturel immatériel en 2013. Dans ces conditions, le ministère de l'Agriculture, des Forêts et de la Pêche promeut la diffusion des connaissances et des compétences concernant la nourriture japonaise dans le cadre de formations des cuisiniers et des restaurateurs japonais à l'étranger, ainsi qu'en établissant un système de qualifications par niveau pour la qualité, les aspects sanitaires et le savoir-faire. 
Ce sont là quelques-unes des mesures de planification de la culture alimentaire japonaise qui sont promues depuis 2015 par le ministère de l'agriculture, des forêts et de la pêche au travers du plan «La nourriture japonaise attrayante; plan en 10 actions». 7

\section{3 «Nul n'est prophète en son pays»: les différents programmes de défense}

La culture alimentaire du Japon est maintenant dans une période de grand changement. Il y a de moins en moins de gens qui cuisinent eux-mêmes à cause des évolutions contemporaines des modes de vie et du développement de l'industrie alimentaire (services de restauration, livraisons à domicile, plats à emporter, fast-food, etc.). Ceci a incité le gouvernement à introduire des mesures législatives pour rééduquer les citoyens sur les traditions et la nourriture, et surtout pour leur rappeler les valeurs nutritives du riz. L'éducation alimentaire, sous l'égide de différents ministères, consiste à élever les enfants - et même les adultes - pour qu'ils puissent maîtriser le choix des aliments qu'ils consomment et avoir des connaissances sur la nourriture à travers une variété d'expériences et de pratiques d'une alimentation saine.

Le 10 juin 2005, la loi fondamentale de l'éducation sur la nourriture (Shokuiku Kihon $H \bar{o}$ 食育基本法 ${ }^{8}$ ) a été votée. Shokuiku (食育) veut dire éduquer ou donner la connaissance (育) sur la nourriture (食). L'objectif de la loi est de cultiver un esprit et un corps sains tout au long de la vie par l'éducation alimentaire. Le shokuiku est une action à laquelle le Premier ministre et 12 ministères participent et qui relève de la sécurité publique au Japon. Il n'y a aucune loi semblable dans le monde entier.

(a) La promotion du ministère de l'Éducation, de la Culture, du Sport et des Sciences (Monbu kagaku shō文部科学省):

Ces dernières années, les problèmes concernant la santé des enfants sont devenus de plus en plus graves: mauvaise nutrition, obésité, pratiques d'amaigrissement comme la suppression du petit déjeuner, etc. En outre, il est considéré comme important de comprendre la culture alimentaire, de même que le travail qui est fait pour cultiver les aliments et connaître les régions se spécialisant dans la nourriture japonaise traditionnelle. Comme les enfants peuvent assimiler une bonne connaissance et acquérir des habitudes alimentaires souhaitables, l'éducation alimentaire à l'école est importante. Le ministère de l'Éducation, de la Culture, des Sports, le ministère des Sciences et de la Technologie essaient ainsi d'améliorer l'orientation sur la nourriture, notamment à travers les repas scolaires en utilisant des produits adaptés et du riz.

\footnotetext{
${ }^{7}$ Nihonshoku miryokuhasshin akushon puran 10 日本食魅力発信アクションプラン 10.

${ }^{8}$ http://elaws.e-gov.go.jp/search/elawsSearch/elaws_search/lsg0500/detail?lawId=417AC1000000063 \&openerCode $=1$.
} 
(b) La promotion du ministère de l'Agriculture, des Forêts et de la Pêche (Nōrin suisan sho 農林水産省):

Le ministère de l'Agriculture, des Forêts et de la Pêche offre à la jeune génération l'occasion de penser à la nourriture, d'améliorer son régime alimentaire, et promeut des activités concernant l'agriculture, la sylviculture, la pêche et les industries alimentaires pour encourager les consommateurs à adopter de bonnes habitudes de consommation. Selon une enquête, ${ }^{9}$ qui a été réalisée sur un échantillon de près de 3 millions de personnes afin de comprendre la situation réelle des habitudes alimentaires et de la consommation alimentaire pour les hommes et les femmes de plus de 20 ans, les éléments les plus notés étaient: (i) sauter les repas; (ii) une faible fréquence de la consommation de riz; (iii) manger à l'extérieur (remplacement du repas fait à la maison), consommer des surgelés et de la nourriture instantanée ou en conserve; (iv) ne pas pouvoir faire la cuisine ou suivre de moins en moins fréquemment le «régime de style japonais», soit associer l'aliment de base (riz) avec les plats principaux et des légumes.

Afin d'ajuster l'équilibre nutritionnel, on promeut la reconnaissance des avantages du régime du riz et la pratique du style alimentaire japonais. Ainsi, en mars 2011, le ministère de l'Agriculture, des Forêts et de la Pêche, dans le cadre des initiatives d'éducation alimentaire, a lancé le «plan Maji $\left(\right.$ sérieux $^{10}$ ) de riz» pour inciter les consommateurs à reconsidérer leur relation à la nourriture. En rappelant le discours du ministre de l'Agriculture, des Forêts et de la Pêche, le secrétaire parlementaire Mr.Tanabe ${ }^{11}$ à l'époque, on peut voir que ce projet considère non seulement le rôle important de la prise de conscience de la manière de se nourrir, mais aussi la

\footnotetext{
9 Manger est l'une des activités fondamentales pour les humains. «Manger» n'est rien moins que «vivre».

10 maji est un mot d'argot des jeunes qui pourrait se traduire par l'expression «grave»; il est utilisé comme adverbe et adjectif.

11 Manger est l'une des activités fondamentales pour les humains. «Manger» n'est rien moins que «vivre».

$\mathrm{Au} \mathrm{XXI}^{\mathrm{e}}$ siècle, n'oublions-nous pas que «vivre, c'est manger».

Ne pensons-nous pas que «manger» veut dire «manger (n'importe quoi) pour remplir le ventre?»

Jusqu'à présent, le ministère de l'agriculture, des forêts et de la pêche, afin d'augmenter le taux d'autosuffisance alimentaire, offrait un mouvement présent sous les deux mots d'ordre ou slogans suivants: «Mangeons du riz!» et «bien équilibré, nous allons manger le riz!» pour être en bonne santé.

Cependant, ces pensées-là, tout en les ayant «dans la tête», ne conduisent pas à l'action.

Voilà pourquoi, je crois qu'il est essentiel que nous voulions agir de notre plein gré et non sous la pression parce que c'est nécessaire et non pas à la suite de leur interrogation.

Quel sens «le riz» a-t-il dans votre vie?

Qu'est-ce que le «riz sérieux» veut dire pour vous?

Manger le riz plus lentement.

Manger quelque chose de savoureux pendant la conversation avec des amis ou des collègues.

Cuisiner soi-même pour manger avec une personne très importante.

Nous voulons créer une occasion de penser à «manger un peu plus positivement».

A l'occasion de repenser notre propre repas, nous allons transmettre ces sentiments; les gens qui cultivent le riz pensent comment encore mieux cultiver, les gens qui mangent pensent comment mieux manger, les gens qui vendent pensent comment encore vendre mieux, c'est le sentiment «Maji».

Nous voulons créer un lien sérieux, et pas seulement une relation du «vendeur» à l' «acheteur».
} 
qualité de ce qui est produit, vendu, comme les conséquences des choix alimentaires effectués.

(c) La promotion du ministère de la Santé, du Travail et des Affaires sociales (Kōsēe rōdo shō厚生労働省):

On peut résumer cet effort de la loi par les trois piliers proposés par le ministère de la Santé, du Travail et des Affaires sociales:

(i) Promotion d'un mouvement en faveur de la santé publique;

(ii) Soutien pour éduquer les enfants;

(iii) Communication sur les risques de la sécurité alimentaire.

\subsection{La journée d'Omusubi}

Cette identité japonaise renouvelée autour de la nourriture se crée actuellement grâce à la journée d'Omusubi pour mettre en avant l'importance de manger du riz.

Un séisme d'une magnitude de 6.0 sur l'échelle de Richter de Hanshin-Awaji qui a touché la région de la ville de Kobe le 17 Janvier 1995 a provoqué une pénurie d'alimentation. Les victimes, dans les profondeurs de l'anxiété, ont été aidées par des bénévoles qui faisaient des omusubi (おむすび, syn. onigiri おにぎり), soit des boulettes de riz généralement enveloppées d'une algue nori et souvent de forme triangulaire ou ovale. De l'expérience de ce tremblement de terre, «un mouvement national qui essaie de manger du riz» a commencé. Au sein du Conseil national, le 17 janvier a été défini comme «la journée d'omusubi» afin de ne pas oublier l'importance de la nourriture, surtout du riz, et les bonnes intentions des volontaires. On remarque aussi l'existence de slogans publicitaires autour de cette journée tels que «Je t'aime, toi qui adores le riz», «As-tu déjà vu (mangé) du riz aujourd'hui?» ou encore «L'archipel du riz, tu veux un autre bol de riz?». ${ }^{12}$

\subsection{La relation profonde entre le riz et les Japonais}

Le riz est non seulement un aliment pour les Japonais, mais il est aussi impliqué profondément dans la vie quotidienne par divers rituels et coutumes tout au long de l'année. Les Japonais priaient pour de bonnes récoltes et remerciaient de pouvoir consommer du riz. De nombreux Japonais se sentent également familiers avec un beau paysage de champ de riz. Une variété de festivals et de coutumes concernant la culture du riz existe encore aujourd'hui pour maintenir l'idée que la culture du riz est une tradition essentielle héritée depuis longtemps:

(i) Ninamesaii (新嘗祭) est un rituel pour sacrifier, au dieu, le grain récolté dans l'année. Aujourd'hui, il est devenu un rituel important que l'empereur fait

12 ごはんを食べよう国民運動推進協議会 www.gohan.gr.jp/. 
chaque année le 23 novembre (c'est un jour férié, la journée pour remercier les personnes qui travaillent). C'est en effet une cérémonie très ancienne qui a commencé durant la période Asuka (592-710). Parmi les Ninamesai, celui effectué par l'Empereur après son couronnement est appelé Daijosai.

(ii) Dengaku (田楽) est une tradition artistique qui a été créée durant la période Heian (794-1185). Elle a été développée à partir de Taasobi (prière pour une bonne récolte avant de planter le riz). Elle est présentée aujourd'hui dans une variété de festivals selon la région, avec de la musique et de la danse qui représentent chaque étape de la culture du riz.

(iii) Otaue (お田植), hanataue (花田植え) sont associées aux étapes de la culture du riz; taue (la plantation du riz dans les champs) est particulièrement importante. Quand arrive la saison de la plantation du riz dans les champs, l'événement traditionnel appelé otaue ou hanataue a lieu dans de nombreuses régions afin de prier pour la croissance et la bonne récolte du riz. Les femmes saotome en kimono bleu avec une ceinture rouge plantant le riz dans les champs est un spectacle à voir.

(iv) Natsumatsuri (夏祭り), akimatsuri (秋祭り) sont des festivals d'été et d'automne qui se déroulent dans tout le pays pour célébrer une bonne récolte ou la gratitude pour la moisson au dieu des champs de riz (Tanokami). Ils marquent le temps et l'espace, la vie du quotidien et même les loisirs du Japon, comme pour la discipline sportive du sumo. Ainsi le shiko o fumu (un geste de sumo) exécuté avant l'affrontement, quand les rikishi (lutteurs) lèvent les pieds très haut, est effectué pour chasser le mal de la terre et prier pour une bonne récolte, en tapant ensuite fermement le sol avec les pieds.

\section{Le langage du riz}

\subsection{L'idéogramme 和 (wa)}

Le terme washoku (la nourriture japonaise) se compose de deux idéogrammes: 和 $(w a)$ et 食 $(s h o k u)$. 食 veut dire «manger, vivre, avaler, mâcher, alimentation, vie, etc.». C'est un hiéroglyphe qui représente la nourriture dans un plat, couverte par un couvercle. 和, lui, a beaucoup de sens, tel que «soulagé, tranquille, clair, calme, élastique, paisible, agréable, détente, doux, décontracté, adouci, embaumé, raisonnable, harmonieux, équilibré» et «Japon». Etymologiquement, 和 est un caractère qui est formé par un symbole qui donne le sens et par un symbole qui donne la prononciation. Le côté droit de 和 est un caractère 口 qui veut dire la «bouche»; le côté gauche 禾 est une image qui signifie «des oreilles de riz suspendus sur la pointe». Cette partie est comprise comme «adapter à la voix des gens», ce qui donne le sens «calme, harmonieux». Nous pouvons dire que 和 veut dire que «le Japon» est formé par l'image du riz même du point de vue linguistique. 


\subsection{Termes applicables au riz}

Puisque le mot «riz» est profondément lié au mot «Japon», nous pouvons postuler que la notion de «riz» est traduite dans la langue japonaise, plus que dans toute autre langue, surtout en Europe, sous ses différents aspects.

En effet, selon sa nature, son état ou ses transformations, les caractères utilisés pour exprimer le riz sont différents: le riz avant ou après la récolte, le riz cru ou cuit, etc. Le riz cru après la récolte s'appelle kome (米), mot utilisé en général pour exprimer le riz comme équivalence des mots «riz» en français, «rice» en anglais, ou «ryż» en polonais. Le riz cuit s'appelle gohan (ご飯), qui veut dire également le «repas». Le riz avant la récolte s'appelle ine (稲), mot qui était utilisé autrefois comme prénom pour les femmes.

Le riz sera nommé différemment, et pas uniquement selon son état ou apparence, mais aussi selon certaines situations. Par exemple, au restaurant de sushis, le riz est appelé shari (シャリ), qui veut dire «le riz blanc». Parfois, il est même surnommé ginshari (銀シャリ), «shari en couleur argent», pour souligner que le riz est très blanc. Utilisé seulement dans les restaurants de sushis, shari est un terme créé à l'origine en prenant le son de sarira en sanskrit qui veut dire «le reste», «le corps du mort». On dit que le grain de riz est appelé shari puisqu'il est similaire à l'os de la matière particulaire qui reste après la crémation du cadavre.

Les appellations du riz peuvent varier également selon la génération ou le genre du locuteur. Ainsi, les hommes disent souvent à propos du riz, meshi (飯), qui veut dire également le repas. Les enfants, eux, disent manma (まんま), qui a pour origine le mot «umai (délicieux)». Comme les mots d'enfants sont souvent répétés, «umai, umai» a donné «umauma» puis «manma».

On doit par ailleurs noter que lorsque le riz est consommé dans le cadre d'une cuisine d'origine occidentale, il est généralement appelé raisu (ライス) et mangé avec une cuillère plutôt qu'avec des baguettes, par exemple sous forme de karēe raisu (le riz avec du curry).

\section{3 Étymologies et collocations de kome}

Il existe plusieurs théories sur l'étymologie du mot kome (riz). Tout d'abord, la première hypothèse est qu'il vient du mot komeru (込める), qui veut dire «mettre quelque chose dedans, remplir». Depuis les temps anciens, le riz est utilisé dans divers festivals et cérémonies. Le riz était quelque chose de sacré et les gens pensaient qu'une puissance mystérieuse (la force, le pouvoir magique) était «mise» (komerareta) dedans, donc il fut appelé le kome. La deuxième proposition pour expliquer l'origine du mot est qu'il serait dérivé d'un mot coréen «醸》 signifiant le fermentation du riz (pour faire le sake). La fermentation du riz a été transmise au Japon en provenance de Corée. A ce moment-là, il est dit que le mot kamosu (fermentation) a été formé et introduit dans le japonais, dérivant vers le terme kome. Enfin, la dernière explication serait que «les petits fruits» du mot komi 
(小実, petits fruits) auraient donné le nom kome car les oreilles du riz étaient exprimées par les deux mots «小ko (小, petit) et $m i$ (実, fruit).

L'idéogramme 米 (kome) est un caractère représentant les oreilles du riz. Il est écrit autour de la ligne horizontale six courtes lignes verticales, signifiant les oreilles du riz suspendues. Une autre théorie dit que le caractère montre quatre grains $(\backslash)$ séparés (十, ancienne forme de 七). Le résultat est ainsi la graine proprement dite. Le caractère de «米», lors de la décomposition peut être divisée en «八huit» et «十 dix», et «八huit». Autrement dit, «quatre-vingt huit». Il a été dit que le travail pour le développement du riz a quatre-vingt huit étapes, soulignant que sa culture est compliquée et dure. En outre, au Japon, il existe la coutume dite de beiju pour célébrer les gens âgés de 88 ans.

Par ailleurs, «米» est, tout à fait indépendamment de son sens originel, utilisé pour représenter l'unité de longueur métoru. Cela provient de la translittération du mot français «mètre». Cet idéogramme est également utilisé lors de la représentation des «États-Unis». Il est comme ceux qui sont venus de l' «Amérique» (米mei, mai) au moment de la translittération des États-Unis sur le plan phonétique.

Il y a beaucoup de caractères qui contiennent l'idéogramme «米». Tout d'abord, 粳 (uruchi) représente un type de riz, le «type japonica (non glutineux)» et 糯 (mochigome) veut dire le riz gluant. 糠 (nuka, son de riz), 粽 (chimaki, boulette de riz gluant), 粥 (kayu, bouillie de riz) et糊 (nori, colle) représentent les choses produites à partir du riz. 糖 $(t \bar{o})$ veut dire le sucre. 立 (tatsu, se tenir debout) rajouté à 米 donne 粒 ( $t s u b u$, grains de céréales); idem avec 分 (wakeru, diviser) qui donne 粉 (kona, farine) et量 (ryō, quantité) qui donne糧 (kate, nourriture). En accolant la couleur blanche (白 shiro), on a粕 (kasu), ce qui reste dans la production de sake, mais en accolant la couleur bleue (青 $a o$ ), on a 精 (sei), la vitalité ou l'esprit.

\subsection{Les différentes classifications du riz selon les types}

Le riz japonais peut être classé selon divers critères.

(a) Classification par la composition en amidon

(i) うるち米 uruchi mai (le riz dit non gluant ${ }^{13}$ ) est le riz le plus généralement consommé au Japon. Simplement écrit kome, il désigne en général le riz non gluant. Il a été distingué du riz gluant par rapport à sa composition en amidon. Le riz non gluant est constitué de $15 \%$ à $35 \%$ d'amylose et de $65 \%$ à $85 \%$ d'amylopectine.

(ii) もち米 mochi gome (le riz gluant) est le riz à utiliser lors d'une préparation de gâteaux de riz ou de riz rouge (il est cuit à la vapeur avec des haricots rouges et mangé lors de festivités comme la remise des diplômes à l'université). Il ne comprend pas d'amylose dans l'amidon et ne se compose que d'amylopectine. Il se caractérise aussi par une texture collante par rapport au riz non gluant.

$\overline{13}$ En fait, le riz japonais est gluant, mais il y a des types moins gluants que l'on appelle «non gluants». 
(b) Classification par variétés

(i) ジャポニカ米 Japonica est le riz que l'on mange habituellement au Japon. La plupart du riz cultivé au Japon est la variété japonica. Il a une forme ovale. Après la cuisson, il devient gluant.

(ii) インデイカ米 Indica a une forme allongée. On l'appelle «le riz thailandais» au Japon. $80 \%$ du riz qui est cultivé dans le monde est la variété indica. Peu gluant et squameux, il est adapté à une utilisation telle que dans les recettes de riz pilaf et de types curry.

(iii) ジャパニカ米 Javanica est cultivé en Asie du Sud-Est, comme sur l'île de Java, en Indonésie, et en Europe comme en Italie ou en Espagne. Sa forme est entre le japonica et l'indica. Il se caractérise toutefois par un grain plus grand que pour ces deux types. Le goût est simple et il est approprié pour le risotto et la paëlla.

(c) Classification par buts d'utilisation

(i) 酒米 Sakamai (riz pour le sake) est utilisé comme matière première pour la fermentation. Il est désigné pour l'utilisation dans la fabrication du saké, dont une partie de l'extérieur des grains est coupée. Il est de fait plus grand par rapport au riz ordinaire. Par contre, il ne convient pas pour une autre utilisation (consommation en l'état). Le riz sakamai est régi par la loi et il n'est pas vendu sur les marchés.

(d) Classification selon la transformation du grain

(i) 白米 Hakumai est un état du riz quand le germe (embryon) a été enlevé du riz brun. Ce riz est l'aliment le plus familier des Japonais.

(ii) 玄米 Genmai (riz brun) est l'état avant d'enlever l'enveloppe des grains. En d'autres termes, c'est le stade du produit avant le riz blanc.

(iii) 発芽玄米 Hatsuga genmai est le riz brun (genmai) germé (hatsuga). Si on laisse immergé dans l'eau du riz brun pendant environ un à deux jours à une température constante $\left(32^{\circ} \mathrm{C}\right.$ environ), un bourgeon d'environ $1 \mathrm{~mm}$ sortira.

(iv) 分づき米 Buzukimai consiste à laisser une petite partie du riz brun lors de l'usinage (fraisage) du riz.

(a) 三分づき米 Sanbuzukimai est un riz de couleur brune et son taux en apports nutritifs et en fibres alimentaires reste élevé.

(b) 五分づき米 Gobuzukimai est un stade entre le riz blanc et le riz brun. L'apparence est néanmoins plutôt blanche.

(c) 七分づき米 Nanabuzukimai a une apparence qui ressemble beaucoup au riz blanc, mais il a une valeur nutritive plus élevée.

(v) 無洗米 Musenmai (le riz sans rinçage). Le riz brun est gratté pour faire du riz blanc. De cette façon, on peut faire du riz blanc courant, mais sur la surface de ses grains, il reste encore une couche collante appelée hadanuka. 


\section{La réglementation des appellations}

Aujourd'hui, il y a plus de 300 variétés de riz. Celles qui sont mangées habituellement ont des appellations. L'histoire de la naissance du riz, les mots pour décrire la région de naissance, etc. sont pris en compte lorsqu'on donne un nom au riz.

Il y a des appellations qui s'écrivent en «hiragana» et en «katakana». ${ }^{14}$ Avant, il y avait des règles strictes pour l'écriture d'une appellation. Les appellations de variétés de riz que les préfectures développaient étaient écrites en «hiragana». Par contre, les appellations de variétés de riz que le gouvernement et le pays développaient étaient écrits en «karakana». Il avait également été décidé que les appellations devaient comprendre au maximum six caractères. Un changement de règles est visible depuis 1991, quand une variété cultivée à la «station expérimentale agricole de Furukawa», institution nationale, a été nommée «ひとめぼれ» (hitomebore) en hiragana. Les régions suivirent ce modèle en libéralisant les règles de fonctionnement de leurs propres appellations. Ainsi, elles sont devenues plus singulières et plus libérales. Certains éléments relatifs aux développeurs et aux producteurs sont indiqués en bref dans l'appellation du riz. Il est important de décider de l'appellation, qui influence l'image des consommateurs sur ce riz. Nous présentons ci-dessous quelques exemples de cette diversité et montreront les grands traits qui caractérisent ces noms.

(a) Les appellations données à partir de noms régionaux et de noms de lieux

(i) あきたこまち Akita komachi

Ce riz est produit dans la préfecture d'Akita (Akita-ken), dans la ville d'Ogachi. Onono Komachi (poétesse japonaise de waka (poésie japonaise) de l'époque Heian) est née dans cette ville, donc l'appellation est prononcée comme son nom.

(ii) コシヒカリ Koshi hikari

Koshi veut dire des régions koshi (etsu) et hikari veut dire «la lumière», pour désigner la région Hokuriku. Ce riz a commencé à être produit à partir de 1956 dans la préfecture Niigata, dans la ville d'Uonuma, qui est très connue pour la culture du riz.

(iii) つがるロマンTsugaru roman

Ce riz a été produit par le croisement de deux variétés, Fukei 141 go et Akita komachi. Il a été nommé avec les termes passion et romantisme (le mot roman est lié au mot «romantisme») par les producteurs de riz de la région Tsugaru.

(iv) いわてっこIwatekko

Dans la préfecture d'Iwate, lors de la naissance de ce riz, l'appellation a été demandée au public. L'appellation devait exprimer la richesse de la nature et la fierté des producteurs. Plus de 8 personnes ont proposé Iwatekko (Iwate, le nom de la préfecture et $k k o$ 子, les enfants).

(v) つくしろまんTsukushi roman

${ }^{14}$ Deux systèmes de caractères phonétiques employés en parallèle. 
C'est l'ancien nom de la province de Kyūshū (tsukushi), associé au mot roman (romantisme).

(b) L'appellation donnée à partir du numéro d'un endroit de culture et d'onomatopées

(i) きらら397 Kirara 397

Cette appellation a été donnée sur proposition des habitants d'Hokkaido. Le kirara signifie une «étincelle» et «397» représente le nombre de parcelles cultivables de riz sur place.

(c) L’appellation donnée à partir du type de riz

(i) ササニシキ Sasanishiki

Sasanishiki est né en 1961 dans la préfecture Miyagi par le croisement des variétés « Sasa shigure» et «Hatsu nishiki». Il est aussi considéré comme ayant son origine du nom de la chanson folklorique さんさん時雨Sansan shigure.

(d) Les appellations données dans le dialecte utilisé dans la région

(i) おいでまい Oidemai

Oidemai est un dialecte de la province de Sanuki, au nord-est de l'île de Shikoku, qui est aujourd'hui la préfecture de Kagawa. Oidemai signifie «bienvenue». Il semble avoir été donné avec l'espoir que les gens viennent manger dans la préfecture de Kagawa.

(ii) ちゅらひかり Chura hikari

Chura, le nom du dialecte d'Okinawa, veut dire la «beauté» et hikari «la lumière». Cette appellation laisse entendre que l'avenir de ce riz est brillant.

(iii) めんこいな Menkoina

Dans le dialecte de la région d'Akita, lieu de production de ce riz, menkoi veut dire «mignon» ou «charmant». Cette appellation semble avoir été donnée avec le souhait que le riz soit aimé par les consommateurs.

(e) Les appellations données à partir de la forme et de la couleur du riz

(i) おぼろづきOborozuki

Oborozuki veut dire «la lune voilée», par analogie avec la couleur de ce riz.

(ii) キヌヒカリ Kinuhikari

Ce nom est l'association de kinu «la soie» et de hikari «la lumière».

Cette appellation a été donnée en référence à l' apparence du riz blanc, brillant comme de la soie, après la cuisson.

(iii) ミルキークイーン Mirukī kuīn

Mirukī, de l'anglais milky est ici associé à kuīn, de l'anglais queen. Cette appellation a été donnée parce que la couleur de la surface du riz est laiteuse et que cela le rend beau comme une princesse. 
(f) L'appellation donnée à partir des sentiments et des désirs du producteur

(i) 元気つくし Genki tsukushi

Ce nom s'apparente à l'image du riz cultivé, sain et fort, pendant le temps chaud sur l'île de Kyūshū et celle d'un produit délicieux qui donne de l'énergie aux gens. Tsukushi est le nom de l'ancienne préfecture de Fukuoka.

(g) L’appellation donnée à partir d'onomatopées

(i) ゴロピカリ Goro pikari

Goro est l'onomatopée qui signifie que le tonnerre gronde fortement. Pikari est l'onomatopée qui veut dire qu'un éclair apparaît. Ce riz est cultivé dans la préfecture de Gunma, qui est connue pour la foudre qui y tombe.

(ii) ふつくりんこ Fukkurinko

Fukkuri est l'onomatopée qui veut dire «être potelé». Cette appellation a été choisie par le public. Fukkuri donne une image appétissante.

(h) L'appellation donnée en utilisant des proverbes

(i) 青天の霹靂 Seiten no hekireki

Seiten no hekireki veut dire un coup de tonnerre. Il a été nommé en souhaitant que le riz devienne un produit vivant d'Aomori comme le ciel où il fait apparâ̂tre des éclairs. 《霹靂》 est composé par deux caractères hors de la liste de 2136 jōyōkanji (caractères prescrits pendant l'éducation obligatoire).

(i) L'appellation donnée par les noms d'animaux et de choses qui ont de l'importance localement

(i) 森のくまさん Mori no kumasan

Mori no kumasan veut dire «un ours dans la forêt». L'appellation a été choisie par le public. La préfecture de Kumamoto est connue pour ses forêts (mori) où il y a des ours (kuma). Le caractère san (さん), placé à la fin d'un nom, veut dire «Monsieur ou Madame». Néanmoins, à la fin d'un objet, san (産) indique l'endroit où il a été produit. Il y a donc ici deux sens.

Les appellations mentionnées ci-dessus peuvent être divisées en deux types: celles qui tiennent leur origine de la variété du riz considérée et les autres, comme celles créée par les localités et les habitants. Ces deux types d'appellations ont différentes protections juridiques, décrites ci-dessous.

(a) Les appellations qui viennent de la variété du riz

Ce type d'appellations est par exemple Koshihikari, Sasanishiki, Kirara397, Hitomebore, etc. Mais qu'entend-t-on exactement par la définition de «l'appellation de la variété»? Cela désigne les cas où les appellations de la variété en question sont de deux types: le premier cas, lors de l'achat et de la vente 
des graines de riz (les semences) et des plants aux agriculteurs; le deuxième cas, lorsque les agriculteurs vendent le riz aux consommateurs comme produit de consommation.

Le schéma ci-dessous [3] montre l'écoulement des ventes de semences et de plants de riz (A) et la production et la vente du riz comestible (B). Dans la partie A du schéma, les appellations veulent dire les noms des semences et des plants de riz; dans la partie B, les appellations veulent dire les noms de production. Si on utilise les appellations comme les noms des semences et des plants, elles sont réglementées et protégées par la «Loi sur les semences et les plants» (種苗法 Shubyōhō), ${ }^{15}$ qui définit les règles de protection des origines et de création de nouvelles variétés de plantes. Ceux qui ont créé de nouvelles variétés de plantes (fleurs et produits agricoles, etc.) en les enregistrant officiellement acquièrent le droit exclusif de les cultiver. La loi sur les semences actuelles a été modifiée en 1998, au regard de la Convention internationale pour la protection des obtentions végétales, elle-même modifiée en 1991 (abréviation: Convention UPOV).

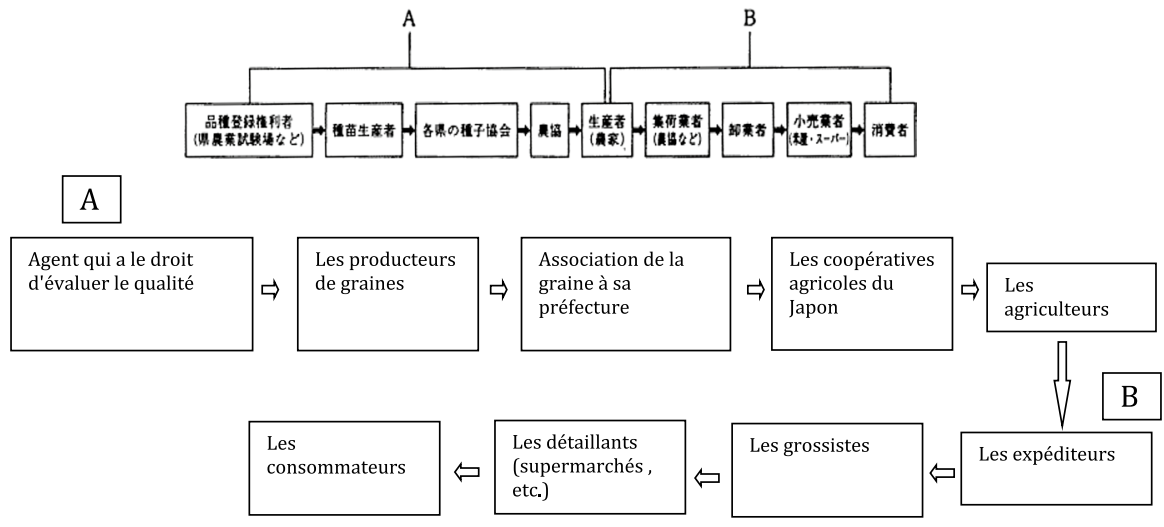

(b) Les appellations de marque originale

Les appellations du riz peuvent être nommées par les agriculteurs, les coopératives agricoles ou les distributeurs de chaque localité, comme par exemple: Mirukī kū̄n ( ミルキークイーン), Pāru raisu (パールライス), Mori no kumasan (森のくまさ ん), etc.

Ces appellations peuvent s'enregistrer comme shōhyō 商標 (marque) et sont alors protégées par le droit des marques car elles ne sont pas soumises à l'enregistrement au regard de la loi sur les semences, mentionnée ci-dessus.

La figure ci-dessous [3] montre des exemples de riz, avec leurs numéros d'enregistrement, qui ont été établies comme des marques. Elles sont soumises aussi cependant à la loi sur la traçabilité.

\footnotetext{
15 http://elaws.e-gov.go.jp/search/elawsSearch/elaws_search/lsg0500/detail?lawId=410AC0000000083 _20171126\&openerCode $=1$.
} 


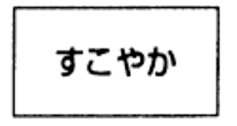

商组登睩第1004042号

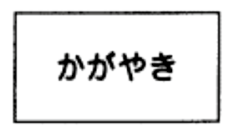

商標登镍第1140767号

すこやか
sukoyaka

$\mathrm{N}^{\mathrm{o}}$

d'enregistrement

de la marque

commerciale

1004042

かがやき
kagayaki

$\mathrm{N}^{\mathrm{o}}$

d'enregistrement

de la marque

commerciale

1140767

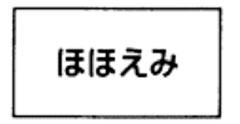

商掼登绿第1004043号

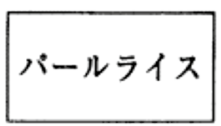

商僄登解第1204268号

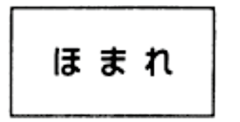

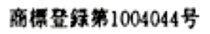

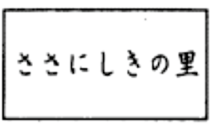

商標登绿第1253663是

\section{COOP\&COOP}

商標登録第1004045号

タマノヒカリライス

t:1000.nsut

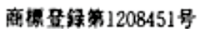

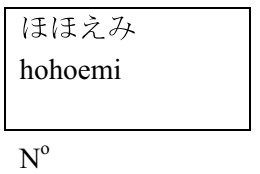

d'enregistrement de la marque commerciale 1004043

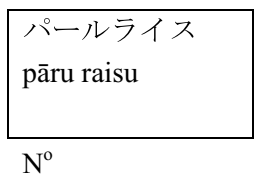

d'enregistrement de la marque commerciale 1204268

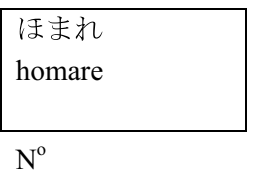

d'enregistrement de la marque commerciale 1004044

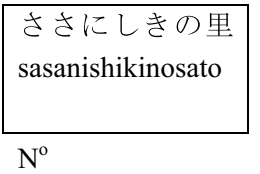

d'enregistrement de la marque commerciale 1253663 \begin{tabular}{|l|}
\hline COOP\&COOP \\
\hline $\mathrm{N}^{\mathrm{o}}$ d'enregistrement
\end{tabular}

de la marque commerciale 1004045

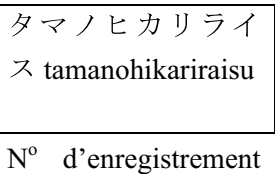

de la marque commerciale 1208451

\section{Cadre juridique}

Nous avons évoqué la loi sur la traçabilité concernant le riz, mais ce n'est ni la seule, ni la plus ancienne, loin de là, à avoir été créée pour règlementer le marché du riz au Japon. Déjà au XVIII ${ }^{\mathrm{e}}$ siècle, ${ }^{16}$ on peut noter un intérêt croissant à vouloir réguler ce marché.

\footnotetext{
16 Nous voudrions faire remarquer qu'en analysant l'aspect linguistique de la loi sur le contrôle des aliments appelée «shokkanhō», promulguée le 21 février 1942, nous avons relevé que celle-ci avait été écrite en Kanji et Katakana. Ce style d'écriture s'appelle «Kanamajiri bun». Les lois ont commencé à être promulguées au Japon sous l'ère Meiji. A cette époque-là, on utilisait ce style pour les documents officiels et c'est pour cela que lors de la promulgation des lois, même aujourd'hui, ces lois sont écrites dans ce style caractéristique pour les documents officiels. Pour cette raison, la loi actuelle japonaise, aussi bien le Code civil que le Code pénal, ont été écrits dans ce style au moment de leur promulgation. (Actuellement, la plupart des codes qui ont été écrits dans ce style sont réécrits en hiragana et kanji.). A ce moment-là, elle était écrite dans un style littéraire (定ム Sadamu, スベシ Subeshi, 目的トス Mokutekitosu etc.).
} 


\subsection{Le droit ancien: le riz et la loi}

\section{L'intervention du gouvernement dans la distribution alimentaire (avant et après la Première Guerre mondiale jusqu'à la Seconde Guerre mondiale)}

Avant la Première Guerre mondiale, nous pouvons citer trois dates qui jalonnent la création d'institutions liées avec la gestion du riz. Tout d'abord, en 1730, le centre d'opération à terme (de distribution) du riz a été fondé. À l'époque, il fut mis en place à Osaka, qui était le centre du commerce du riz. Ensuite, en 1886, le marché du riz de Tokyo a été mis en place. Un marché du riz, en tant que produit concret à vendre, a été ouvert à Tokyo. Le riz pour la consommation des habitants de Tokyo était vendu sur ce marché; il devint alors la base pour le commerce du riz. Enfin, en 1908, le marché de Tokyo a été réorganisé en bourse des marchandises du riz. Celle-ci a été mise en place pour les transactions de riz et les échanges de produits de base complets. Des produits agricoles autres que le riz étaient également régis par ce marché.

Après la Première Guerre mondiale, le prix du riz était instable et une loi sur le riz (Beikokuhō 米穀法) fut instituée afin de supprimer les fortes fluctuations des prix. Les achats, les échanges, le façonnage et la provision du riz ont alors été régulés par le gouvernement. Puis, la loi sur le contrôle du riz (Beikokutōseihō 米穀統制法) [4] a été introduite en 1931 pour remédier à la forte différence entre le prix le plus élevé et le prix le plus bas du riz proposé à la vente. Ensuite, la loi sur le riz a été améliorée en 1933. Le gouvernement faisait des achats et des ventes illimités au prix officiel et il exerçait aussi un contrôle sur l'importation et l'exportation du riz.

En 1936, une loi complémentaire à celle de contrôle du riz (Beikokujichikanrihō 米穀自治管理法) a été promulguée. Plutôt que le gouvernement, des acteurs autonomes, tels que les producteurs, ont été autorisés à prendre des mesures pour contrer les excès de production du riz. Le gouvernement obligeait auparavant les producteurs à stocker le riz et il ne permettait pas la libération de son stockage si le prix n'augmentait pas de $10 \%$ au-delà de la norme du prix le plus bas. [5: 757-762]

Le contrôle du riz excédentaire était fait par un Comité de gestion qui imposait des économies d'une certaine quantité déterminée de riz. Les syndicats de l'industrie, en tant que représentants du gouvernement, contrôlaient le riz excédentaire si le prix descendait au-dessous du prix le plus bas. En ce temps-là, puisque l'industrie lourde était développée pour l'expansion militaire et représentait la majorité de la valeur totale de la production industrielle, le gouvernement poursuivit une politique de réduction des disparités de productivité entre l'agriculture et l'industrie afin de parvenir à un approvisionnement alimentaire stable. Cela voulait dire maintenir un prix élevé du riz en faisant correspondre l'offre et la demande, mais cela suscitait la colère des commerçants. Ces lois ayant donné pour résultat une forte baisse du volume des transactions d'échanges de riz, la bourse des marchandises de riz à Tokyo (Tōkyō Beikokushōhin Torihikisho 東京米穀商品取引所) est devenue de fait obsolète dès 1939 et a été remplacée par une société anonyme semigouvernementale (Nihon Beikoku Kabushikigaisha 日本米穀株式会社) en guise d'alternative.

Les ventes de riz à terme ont été supprimées. L'exportation de riz à Taiwan, qui était un facteur de baisse des prix au Japon, a été gelée par le gouvernement. 
Le mécanisme de perception étant une autorité centralisée, le contrôle par le gouvernement a été renforcé par l'exécution du règlement des ventes de riz et le système de son rationnement. Les grossistes et les détaillants de riz étant autorisés par la réglementation et la pression syndicale, le contrôle par le gouvernement s'exerçait jusqu'à la distribution et la vente de riz. L'origine de ce système de contrôle du riz (système de contrôle du riz par le gouvernement) a réellement commencé avec la fondation de la société Beikoku Kabushikigaisha.

\subsection{La loi Shokuryōkanrihō (食糧管理法) sur le contrôle des aliments jusqu'à son abolition}

La loi sur le contrôle des aliments appelée Shokuryōkanrihō (食糧管理法) a été promulguée le 21 février 1942 et supprimée en 1995 lorsque la loi sur la législation alimentaire, Shokuryōhō (食糧法) ${ }^{17}$ a été adoptée. Pour prouver l'importance du rôle de l'état, à partir de cette loi promulguée en 1942, on peut souligner la présence du mot gouvernement. Composée de 57 articles au total, l'article 1 précise «le but» de la loi et son article 2 en présente «la définition». Les articles qui suivent, de 3 à 5, traitent de l'achat du riz par le gouvernement, puis de son importation et de son exportation (le transport), de son stockage, de son échange. Le traitement du riz relève des articles 6 à 9, sa tarification et sa taxation des articles 10 à 13, et la société alimentaire centrale et la société alimentaire locale des articles 14 à 28). Diverses dispositions et la partie répressive (articles 29-43) complètent la loi, avec des dispositions complémentaires (articles 44-57).

L'article 1 stipule:

Cette loi a pour objectif que le riz et le blé, qui sont des aliments de base, jouent un rôle important; en plus, compte tenu du fait qu'ils occupent une position importante en tant que produits agricoles, nous visons à la stabilité de l'offre et de la demande et à un prix stable de l'aliment, par les mesures sur la distribution propre des producteurs de riz aux consommateurs, ainsi que par des mesures nationales globales pour les achats, l'importation et la vente d'aliments de base par le gouvernement; nous devons contribuer à la stabilité de la vie des gens et de l'économie dans l'intérêt national.

Au total, le mot «gouvernement» apparaît 38 fois, comme par exemple dans les articles concernant la société alimentaire centrale ou la société alimentaire locale:

政府八特二必要アリト認ムルトキハ (Lorsque le gouvernement admet qu'il est particulièrement nécessaire,);

政府之习監督ス: (Le gouvernement veille.);

Il y a beaucoup de phrases qui commencent par le sujet «le gouvernement» (政府 八) terminant à la fin avec le prédicat de «prescrit» (定ム), ou avec le complément

\footnotetext{
${ }_{17}$ Officiellement Shuyōshokuryōno jukyū oyobi antei ni kansuru hōritsu (主要食糧の需給及び価格の 安定に

関する法律)：http://elaws.e-gov.go.jp/search/elawsSearch/elaws_search/lsg0500/detail?lawId=406AC 0000000113\&openerCode $=1$.
} 
«par les dispositions des Etats» (定ムルトコロ). Nous retrouvons ceci dans les articles $4,5,6,7,9,10,12,20$, c'est-à-dire 8 articles au total.

Avec cette loi, un prix officiel a été fixé, autre que celui de la valeur de marché déterminée pour la quantité d'orge non-noble, d'orge, de blé, mais aussi du riz. Le gouvernement faisait alors en sorte que les aliments soient fournis aux consommateurs sur la base du rationnement de riz. Il n'était pas permis de le distribuer d'une autre façon. Toutefois, en dépit du risque de punitions ou d'amendes, il y avait des marchés noirs.

Le riz et les aliments de base étaient ainsi contrôlés dans le pays, en assurant un approvisionnement stable. Le canal de distribution était limité, du producteur au gouvernement, et du gouvernement aux consommateurs. Le gouvernement obligeait les producteurs à lui vendre leur riz. Les vendeurs en gros et de détail étaient considérés comme des agents de la mise en œuvre d'un «système de distribution» équitable et efficace par le gouvernement.

Après la Seconde Guerre mondiale, l'approvisionnement alimentaire a été réduit. En plus, à cause du mauvais temps, la production agricole s'est dégradée. En 1946, le gouvernement a donc décidé l'augmentation des prix du riz.

La situation des approvisionnements alimentaires était extrêmement grave, au point que les habitants ont manifesté au niveau national contre la famine ${ }^{18}$ dans le parc Hibiya le $1^{\text {er }}$ novembre 1945. Le 17 février 1946, pour faire face à cette situation, le gouvernement prit des mesures extraordinaires d'urgence alimentaire en promulguant une ordonnance sous la forme d'un édit d'urgence, qui exhortait à une hausse des prix du riz. Mais la grande majorité des agriculteurs éprouvait déjà de la méfiance envers le gouvernement. Dès lors, le 3 mars, alors que l'ordonnance sur le contrôle des prix du riz était promulguée, le niveau de vie des Japonais était extrêmement faible à cause de l'inflation. Le 19 mai, la protestation de la population eut lieu en face de la place du palais impérial; environ 25 millions de personnes, à l'initiative des membres des syndicats, manifestèrent contre la pénurie d'alimentation. ${ }^{19}$

A la faveur d'une grande récolte en 1955, le taux d'autosuffisance en riz a atteint $100 \%$ et des excédents ont commencé à apparaître. A partir de 1969, la loi autorisait à ce que seulement le riz de haute qualité soit autorisé à la vente directe aux grossistes sans passer par le gouvernement. Par celle-ci, le prix du riz a en quelque sorte été libéralisé. Cependant, il a augmenté avec l'inflation et un marché noir est apparu. En conséquence, dans les années 1980, on estime qu'il y avait plus d'un milliard de yen de déficit dans le contrôle des aliments, soulignant que le système ne fonctionnait plus [4].

\footnotetext{
18 餓死対策国民大会.

${ }^{19}$ Connu sous le nom de Shokuryōmē dē 食糧メーデー, ce jour-ci fut considéré comme la fête de la Nourriture et était aussi important que le $1^{\mathrm{er}}$ mai, la fête du Travail.
} 


\subsection{Le droit contemporain: Shokuryōhō (loi concernant la stabilité de l'offre et de la demande et le prix des aliments de base)}

La loi sur le contrôle des aliments de 1942 a été supprimée à cause des deux événements suivants:

(i) L'émeute de 1993 pour protester contre le manque de riz. A cause d'un été froid, il y eut moins de riz produit, suscitant une critique sur la vulnérabilité du système de contrôle des aliments. Pour cette raison, le gouvernement apporta des améliorations à la loi, de manière à être en mesure de mieux réguler le système de vente du riz.

(ii) Les importations de riz. En 1995, suite à l'Uruguay Round ${ }^{20}$ sur l'agriculture, le riz a commencé à être importé de pays tels que les Etats-Unis (principalement pour l'aide à d'autres pays; il n'était pas utilisé pour la consommation au Japon).

Shokuryohō (食糧法), ${ }^{21}$ le droit actuel concernant la stabilité de l'offre et de la demande et le prix des aliments de base, se caractérise par le même souci de stabilité alimentaire que dans les mesures précédentes prises par les autorités japonaises. Ainsi, le 24 juin 1999, fut votée la loi sur la stabilité de l'offre et de la demande et le prix des aliments de base. Amendée en 2004, la loi sur le contrôle des aliments fut abolie, et la nouvelle législation sur la révision partielle de la loi concernant la stabilité de l'offre et de la demande et le prix des aliments de base $\left(2004\right.$, loi $\mathrm{n}^{\circ}$ 103 ) est entrée en vigueur. Avec cette loi, les gens peuvent désormais vendre le riz à n'importe qui et les agriculteurs peuvent le distribuer sans limitation de quantité.

- Chapitre 1, Dispositions générales (articles 1-3)

- Chapitre 2, Les mesures pour stabiliser l'offre et la demande et le prix du riz

- Section 1: Les lignes directrices de base (article 4)

- Section 2: Les mesures pour assurer une bonne distribution

- paragraphe 1: La politique d'ajustement de la production (article 5-7)

- paragraphe 2: Un mécanisme de soutien d'approvisionnement stable de riz (Articles 8-17)

- paragraphe 3: Centre de formation des prix du riz (articles 18-28)

- Section 3: Les achats et les ventes du gouvernement (articles 29-33)

- Section 4: Importation et exportation effectuées par une personne autre que le gouvernement (Articles 34-36)

- Section 5: Mesures d'urgence (Article 37-40)

\footnotetext{
${ }^{20}$ Le cycle d'Uruguay, plus connu sous le terme d'Uruguay Round, est le dernier et le plus important des cycles de négociations internationales ayant eu lieu dans le cadre de l'Accord général sur les tarifs douaniers et le commerce (AGETAC) entre 1986 et 1994. Le cycle d'Uruguay a abouti aux accords de Marrakech (avril 1994) et a finalement donné naissance à l'Organisation mondiale du commerce (1995).

21 Voir la note 19 pour le titre officiel.
} 
- Chapitre 3: Mesures sur l'offre et la demande et le prix de blé et de l'autre aliment stable principal (Article 41-46)

- Chapitre 4: Dispositions diverses (Article 47-54)

- Chapitre 5: Pénalités (Article 55-62)

(a) il n'y a plus de différence entre le «riz de distribution planifiée» et le «riz de distribution non planifiée»:

Le riz est divisé en «riz de distribution planifiée» et en «riz de distribution non planifiée». Le «riz de distribution planifiée» est divisé en «riz pour le gouvernement» et en «riz de distribution volontaire».

(i) Le riz de distribution volontaire. Une mesure du prix a été déterminée au «centre de formation des prix volontaires de riz de distribution». Le riz est recueilli par les grossistes et est vendu par les supermarchés.

(ii) Le riz pour le gouvernement. Il s'appelle 備蓄 bichiku (stock, magasin). Il est le riz utilisé pour maintenir une certaine quantité de stocks en cas de catastrophe ou de guerre. Ce riz est également vendu dans les supermarchés après stockage en entrepôts pendant un an.

(iii) Le riz de distribution non planifiée. Les producteurs de riz peuvent vendre directement aux consommateurs. Ce riz ne peut être vendu dans les supermarchés.

Aujourd'hui, toutefois, le riz de distribution non planifiée peut être vendu dans les supermarchés. Des magasins traitant plus de 20 tonnes de riz par an peuvent vendre librement le riz. En outre, le riz de la distribution planifiée a également commencé à être vendu au JA ${ }^{22}$ et par les agriculteurs.

(b) La politique de mise en réduction des champs de riz (réduction des surfaces de production) va être supprimée en 2018 (décision du 26 novembre 2013):

La politique de mise en réduction des champs de riz est une politique agricole datant de l'après-guerre au Japon pour ajuster et réguler la production de riz. Fondamentalement, le but de cette politique était de réduire la production de riz en contraignant les agriculteurs à une limitation de la superficie des terres rizicoles. Cependant, le 26 novembre 2013, le gouvernement a décidé d'abolir cette politique, avec une mise en application à partir de 2018.

(c) «Le centre de formation des prix du riz» qui était avant «le centre de formation des prix volontaires du riz de distribution» a été aboli le 31 mars 2011:

Auparavant, la limite du prix le plus bas était contrôlé afin de protéger les producteurs (les agriculteurs), mais maintenant il est décidé par le «prix du marché», par la concurrence. Le prix du riz est actuellement fixé par un indice de «la négociation relative» (la négociation relative est égale au prix fixé par l'équilibre de l'offre et de la demande, entre «les producteurs et les vendeurs»).

(d) Le système de notification pour les distributeurs:

$\overline{22}$ Japan Agricultural Cooperatives (Nōgyō Kyōdō Kumiai 農業協同組合). 
Auparavant, le riz de distribution planifiée était géré à l'échelle de la préfecture et le prix était établi par le «centre de formation des prix de riz de distribution» pour que le riz soit vendu aux grands commerçants. Mais aujourd'hui, toute personne qui vend plus de 20 tonnes de riz et qui en a émis la notification (information sur la quantité de riz qui sera vendue) peut vendre librement son riz.

\subsection{Loi sur la traçabilité du riz}

La loi concernant l'enregistrement des informations relatives aux opérations du riz et à la transmission des renseignements sur l'origine $(2009)^{23}$ exige tout d'abord de nommer le produit cible:

1. Le riz; le riz brun; le riz blanchi, les brisures de riz.

2. Ceux qui correspondent à l'aliment principal: la farine de riz; des préparations à base de farine de riz (y compris la préparation de la poudre de riz); les gâteaux de riz; etc.

3. Le riz cuit: les diverses boîtes à lunch avec le riz; les différents types de boules de riz; les hamburgers de riz; le riz rouge avec des haricots rouges,; le riz cuit; le riz emballé; le riz brun germé; le riz cuit comme le riz sec cuit (y compris les aliments congelés, les aliments préparés en autocuiseur et la nourriture en conserve).

4. Les aliments transformés du riz: gâteaux de riz; boulettes; crackers de riz; saké distillé (焼酎 shochu, 味醂 mirin).

Mais il ne faut pas oublier que l'objectif principal est d'obliger les producteurs et les distributeurs d'enregistrer et de conserver les informations sur l'origine et la distribution du riz.

Il y a deux raisons de l'obligation décrite ci-dessous:

(i) Identifier facilement l'itinéraire de distribution au cas où un problème social serait découvert, comme l'usurpation d'identité de la localité d'origine du riz, etc.;

(ii) Fournir aux consommateurs des informations sur l'origine du riz, etc.

En cas d'incident alimentaire, afin d'identifier rapidement l'origine de la distribution, la loi sur la traçabilité du riz oblige toute personne qui produit ou distribue du riz à opérer un enregistrement de toutes les données. Elle oblige également les producteurs à enregistrer les renseignements d'origine du produit afin de pouvoir en faire mention sur les références des biens vendus, ce qui permet une information transparente des consommateurs au moment de l'achat.

23 米穀等の取引等に係る情報の記録及び産地情報の伝達に関する法律. 


\section{Conclusion}

Ainsi, comme nous l'avons vu, le riz n'est pas seulement une graine cultivée, vendue et consommée au Japon. S'il reste, à la base, un produit économique, le riz japonais n'en est pas moins une représentation symbolique et culturelle indéniable pour ses habitants. Ceci est dû non seulement à l'histoire traditionnelle (légendaire et mythique) du riz au Japon, mais aussi à ses inscriptions dans la langue sous ses différentes formes. Il est également, surtout du fait de sa reconnaissance dans le droit japonais comme patrimoine imprescriptible à conserver et à préserver, juridiquement régulé et soutenu dans sa culture et sa présence pour éviter des pénuries et être maintenu dans la vie des Japonais.

Open Access This article is distributed under the terms of the Creative Commons Attribution 4.0 International License (http://creativecommons.org/licenses/by/4.0/), which permits unrestricted use, distribution, and reproduction in any medium, provided you give appropriate credit to the original author(s) and the source, provide a link to the Creative Commons license, and indicate if changes were made.

\section{References}

1. Braudel, Fernand. 1979. Annales. Histoire, Sciences Sociales 2006/5 (61e année), 1193-1242.

2. Bray, Francesca. 1986. The rice economies: Technology and Development in Asian Societies. Oxford: Basil Blackwell.

3. Ministère de l'Agriculture, des Forêts et de la Pêche. http://www.maff.go.jp 2016, accédé 26 October 2016 .

4. Zenbeihan (portail de l'Entreprise nationale de vente de riz coopérative d'aide mutuelle) http:// www.zenbeihan.com/data/history/, accédé 26 October 2016.

5. Yagi Yoshinosuke. 1936. Beikoku jichi kanri hō no jisshi (米穀自治管理法の實施) Keizai ronsō (經濟論叢).

Publisher's Note Springer Nature remains neutral with regard to jurisdictional claims in published maps and institutional affiliations. 